\title{
Impact of brachytherapy technique (2D versus 3D) on outcome following radiotherapy of cervical cancer
}

\author{
Kris Derks, BScl, Jacco L.G. Steenhuijsen, PhD', Hetty A. van den Berg, MD!, Saskia Houterman, PhD², \\ Jeltsje Cnossen, MD, PhD', Paul van Haaren, PhD', Katrien De Jaeger, MD, PhD' \\ 'Department of Radiotherapy, ${ }^{2}$ Department of Education and Research, Catharina Hospital, Eindhoven, The Netherlands
}

\begin{abstract}
Purpose: The purpose of this study was to analyze the effect of 2D conventional brachytherapy (CBT) compared to 3D MRI-guided brachytherapy (IGBT) with and without the use of interstitial needles on local control, overall survival, and toxicity in patients treated for cervical cancer with radiation or chemoradiation.

Material and methods: A retrospective analysis was performed of biopsy-proven FIGO IB-IVA cervical cancer patients, treated with primary radiation or chemoradiation, followed by brachytherapy (BT) between January 1997 and July 2016. Endpoints were local control, overall survival, and toxicity.

Results: Of 126 patients included, 35 have been treated with CBT, 31 with IGBT without needles (IC), and 60 with IGBT with needles (ICIS). External beam radiotherapy (EBRT) had mostly been delivered concurrently with chemotherapy (weekly cisplatin). Overall local control was $93 \%$ after 1 year, and $88 \%$ after 3 years. Overall 3-year survival was $75 \%$, and 5-year survival was $66 \%$. The 3D technique (IGBT cohorts) showed a trend for an improved local control and overall survival $(p=0.05)$ compared to the 2D technique (CBT cohort). A decrease in toxicity was observed from $17 \%$ (2D cohort) to $12 \%$ (3D cohort). The use of interstitial needles was associated with a higher high-risk clinical target volume (HR-CTV) dose (11.3 Gy vs. $9.9 \mathrm{~Gy})$ and a lower $\mathrm{D}_{2 \mathrm{cc}}$ bladder dose (10.9 Gy vs. 14.7 Gy, both $\left.p<0.01\right)$.

Conclusions: In cervical cancer treatment, the use of a 3D brachytherapy technique (MRI-guided with or without interstitial needles) showed a trend towards an increased local control and improved overall survival with reduced toxicity, compared to the conventional 2D brachytherapy technique. The use of interstitial needles allowed dose sculpting, resulting in delivery of higher doses to the HR-CTV, while reducing radiation doses to organs at risk, such as the bladder.

Key words: brachytherapy, cervical cancer, interstitial needles, MRI-guided brachytherapy.

\section{Purpose}

Chemoradiation, followed by a boost dose delivered by brachytherapy (BT), is the standard therapy for patients with locally advanced cervical cancer, and for patients with early stage cervical cancer who are medically not eligible for surgery. Although BT techniques have evolved over time, the application still requires an experienced team with special skills and equipment. Attempts to deliver the boost dose via an external beam radiotherapy technique (EBRT), such as intensity-modulated radiotherapy (IMRT) instead of BT, have resulted in worsening of survival by $12 \%$ [1]. Hence, boosting via BT, remains the gold standard in the radiation treatment of cervical cancer. For many decades, BT was based on orthogonal 2D X-ray imaging. In this approach, the dose is prescribed to a virtual point $\mathrm{A}$, at a fixed distance from the applicator, resulting in a standard pear-shaped isodose configuration.
This technique is referred to as conventional brachytherapy (CBT) [2]. The prescribed dose in CBT is never adapted to the individual patient or tumor anatomy. Therefore, this method results in a potential overtreatment of small tumors and an undertreatment of large tumors. A significant improvement in cervical cancer treatment, resulted from the use of image-guided brachytherapy (IGBT) using computed tomography (CT) or magnetic resonance imaging (MRI) with the applicator in situ, to guide treatment planning [3,4]. MRI is preferred over CT, because it allows more accurate imaging of the tumor extension and soft tissue organs in the pelvic region, thus providing guidance for dose adaptation and, if applicable, dose escalation [5]. The RetroEMBRACE multicenter study showed that MRI-guided BT following chemoradiation in cervical cancer, leads to an excellent three-year local control (LC) of $91 \%$, a pelvic control (PC) of $87 \%$, an overall survival (OS) of $74 \%$, and a cancer-specific 
survival (CSS) of 79\%, with limited severe morbidity [6]. Other studies have also found improvements in two- to three-year LC from 74 to $84 \%$ with CBT to 85 to $97 \%$, with IGBT, with reduced toxicity $[7,8,9,10,11,12,13,14]$.

A further optimization of intracavitary (IC) IGBT was obtained through the use of interstitial needles (IS) during BT application. Several studies showed a better dose distribution to the high-risk clinical target volume (HR-CTV, defined as the cervix plus macroscopic tumor extension), but more significantly, they also showed a reduction of dose in organs at risk (OAR) $[11,14,15,16,17]$.

The aim of this study was to evaluate the impact of 2D BT (CBT) and of 3D BT with and without interstitial needles (IGBT ICIS and IGBT IC) on local control, overall survival, and toxicity in patients with cervical cancer after treatment with primary radiation or chemoradiation. Furthermore, the effect of dose sculpting with interstitial needles was evaluated.

\section{Material and methods}

\section{Patient selection}

This retrospective cohort study comprises data of patients treated between January 1997 and July 2016. Patients were included if they had had a biopsy-proven, stage IB-IVA cervical cancer treated with EBRT to the pelvis ( \pm para-aortic lymph nodes), with or without concurrent chemotherapy or hyperthermia, followed by three fractions of intra-uterine BT. Patients were also included if they have received EBRT at the Institute Verbeeten Tilburg, and were referred to the department for a BT boost.

\section{Study design}

Patients were divided into three cohorts, based on the BT technique used and on the treatment period: 1 . CBT cohort: patients treated with conventional BT between January 1997 and February 2009; 2. IGBT IC cohort: patients treated with MRI-guided BT between March 2009 and March 2012; 3. IGBT ICIS cohort: patients treated with MRI-guided BT between April 2012 and July 2016, with the option of using interstitial needles. If the interstitial needles were inserted, it was done during at least one of the BT applications. Data on patient, tumor, treatment, and outcome were collected from patients' files. Since data collection was a part of routine clinical practice, this study was not subjected to the Dutch Medical Research Involving Human Subjects Act. Hence, approval by the Hospital Ethics Committee was not mandatory for conducting this study.

\section{Treatment characteristics}

FIGO (International Federation of Gynecology and Obstetrics) staging was performed during examination under general anesthesia, and FIGO stages were grouped as follows [18]: group I: FIGO IB1 and IB2, group II: FIGO IIA and IIB, and group III: FIGO IIIA, IIIB, and IVA. Lymph nodes were defined as pathological if they were larger than one centimeter in size along the short axis on MRI/CT, or if they were smaller but showed mor- phological features suspicious for malignancy or showing FDG uptake on PET CT imaging [19]. Chemotherapy consisted of five weekly courses of cisplatin $40 \mathrm{mg} / \mathrm{m}^{2}$ or carboplatin (AUC). If there was a contra-indication for chemotherapy, hyperthermia was administered once a week $[20,21,22]$. Concurrently, EBRT to the pelvis ( \pm para-aortic nodes) was delivered using $3 \mathrm{D}$ conformal or IMRT at a dose of 45 to 50 Gy in 25 fractions, with a sequential or simultaneous integrated boost (varying from 8 to $16 \mathrm{~Gy}$ ) to pathological lymph nodes. After radiation or chemo radiation, all patients received three fractions of intra-uterine BT ( ${ }^{192}$ Ir high-dose-rate).

\section{Procedure: brachytherapy application, contouring, treatment planning, and dose reporting}

The applicator (tandem and ovoids) was inserted by an experienced radiation oncologist (RO). Needles were placed under fluoroscopy. In patients in the IGBT cohorts, the RO and BT technologists contoured the high-risk clinical target volume (HR-CTV), the intermediate-risk clinical target volume (IR-CTV), the bladder, rectum, and bowel, the applicator and, if applicable, the needles. Doses were calculated either with Plato (Nucletron), Flexiplan (Isodose Control), or Oncentra (Nucletron, an Elekta company, Elekta AB, Stockholm, Sweden) based on the TG43 algorithm [23]. For the CBT cohort, the dose distributions were based on the standard loading of the tandem and the ovoids, depending on the size and angle of the applicator. For the IGBT cohorts, dose distributions were optimized by the RO and a medical physicist, according to the Gyn GEC-ESTRO guidelines $[24,25,26,27]$. The dose volume histogram (DVH) parameters used for optimization and approval were $\mathrm{D}_{2 \mathrm{cc}}$ (minimal dose to the most irradiated 2 cc of an OAR) and $D_{90}$ or $\mathrm{D}_{95}$ (dose to $90 \%$ or $95 \%$ of a target). For all patients in all cohorts, the treatment plan was approved by the RO and the medical physicist. The total dose applied to the tumor and OAR was calculated as the sum of the planned total dose of EBRT, and the sum of the planned doses in each of the three BT sessions (using the HR-CTV $\mathrm{D}_{90}, \mathrm{D}_{2 \mathrm{cc}}$ bladder and rectum). The equi-effective dose was calculated using an $\alpha / \beta$ of $10 \mathrm{~Gy}$ for the tumor (EQD2 $\left.{ }_{10}\right)$, and an $\alpha / \beta$ of $3 \mathrm{~Gy}$ for OAR (EQD2 ${ }_{3}$ [ [25]. Given the proximity of the EBRT PTV to the bladder and rectum, and the $99 \%$ coverage of the PTV in the treatment plan, it was assumed that the $\mathrm{D}_{2 \mathrm{cc}}$ OAR dose equals the planned EBRT dose.

\section{Follow-up}

Tumor response was evaluated 8 to 10 weeks after the last BT session, with clinical examination, pelvic MRI (since 2011), and PET-CT. In case of uncertainty, clinical examination was performed under anesthesia with biopsies and a repeat MRI or PET-CT. Follow-up was performed alternately by a gynecologist and a radiation oncologist, and ended after a 5-year disease-free survival.

\section{Endpoints}

The primary endpoint was local control, defined as the absence of disease in the cervix, upper vagina, and 
parametria on clinical examination, imaging and biopsy (if performed). The secondary endpoints were overall survival, defined as death from any cause, and toxicity, defined as an adverse event grade 3-4 using the Common Terminology Criteria for Adverse Events [28]. In addition, the result of dose sculpting by means of interstitial needles was evaluated for patients treated without needles in the first fraction, and with needles in the second fraction of BT by comparing HR-CTV $\mathrm{D}_{90}$, IR-CTV $\mathrm{D}_{90}$, $\mathrm{D}_{2 \mathrm{cc}}$ bladder and rectum.

\section{Statistics}

Patient and tumor characteristics were described with a median and range (defined as the difference between minimum and maximum), and differences were determined using a $\chi^{2}$ test. LC was analyzed at 1 and 3 years. These time points were selected because local recurrences rarely occur after 3 years [12]. LC and OS were calculated from the date of biopsy-proven disease to the date of event or last follow-up. Patients lost to follow-up were censored at the time of last follow-up. The time-to-event analyses were computed using the Kaplan Meier method and the log rank test. Differences in dose fractions were calculated using a paired two-sample t-test. Analyses were performed using SPSS ${ }^{\circledR}$ version 21.0 for Windows (SPSS Inc. IBM company, Chicago, USA). Results with a $p$-value of less than 0.05 were considered significant.

\section{Results}

\section{Patient and treatment characteristics}

A total of 147 patients were registered in the study. Twenty-one patients were not eligible for this study for diverse reasons, leaving 126 patients for the analysis (Figure 1). Twenty-five patients were referred from the Institute Verbeeten. Of the total study population, 35 patients were treated with CBT, 31 with IGBT IC, and 60 with IGBT ICIS (Figure 1). A significant difference was seen with higher median age in the CBT cohort compared to the IGBT cohorts $(p=0.02$, Table 1$)$. Some variation was observed in lymph node involvement $(p=0.17)$ and in FIGO stage distribution $(p=0.19)$, showing more nodal involvement and more advanced FIGO stages in the IGBT cohorts than in the CBT cohort (Table 1). Over time, an increase in the use of PET-CT and chemoradiation was observed, with a statistically significant difference between the three cohorts $(p<0.001)$. In each cohort, hyperthermia was administered concurrent with external beam radiotherapy in one patient because of a contra-indication for chemotherapy (Table 1). The treatment groups were comparable with respect to tumor histology $(p=0.60)$. For one patient in the IGBT ICIS cohort, MRI showed cervical cancer, but despite repeated biopsies, a carcinoma could not be proven. Over time, an increase of EBRT dose (45-50 Gy) was observed, parallel with the shift in EBRT technique from 3D CRT to IMRT $(p<0.001)$ (Table 1). Interstitial needles were used in $82 \%(49 / 60)$ of the patients in the IGBT ICIS cohort (Figure 1). In the other 11 patients, the additional use of needles was not expected to improve the dose distribution.

\section{Outcomes}

The median follow-up (range) for the CBT, IGBT IC, and IGBT ICIS cohorts was 44 (6-166), 50 (7-97), and 20 (5-56) months, respectively.

\section{Local control}

Local control at three years was achieved in 113 of the 126 patients. Of the 13 patients with recurrence, 6 had persistent local disease, while 7 developed a true local recurrence. Overall local control at one year was $93 \%$, and at three years $88 \%$. Local control at one and three years was comparable between the three treatment cohorts: $88 \%$ and $84 \%$ in CBT, $94 \%$ and $90 \%$ in IGBT IC, and $95 \%$ and $86 \%$ in IGBT ICIS (log-rank test Kaplan-Meier curve, $p=0.75$, Figure 2A). Local control at one and three years tended to be lower with increasing FIGO stage: $97 \%$ and $91 \%$ in group I, $93 \%$ and $88 \%$ in group II, and $87 \%$ and $84 \%$ in group III. However, the differences were not statistically significant (log-rank test Kaplan-Meier curve, $p=0.52$, Figure 2B).

\section{Overall survival}

At the time of analysis, 90 patients were alive (71\%), 21 had died from cervical cancer, and 15 from other diseases,

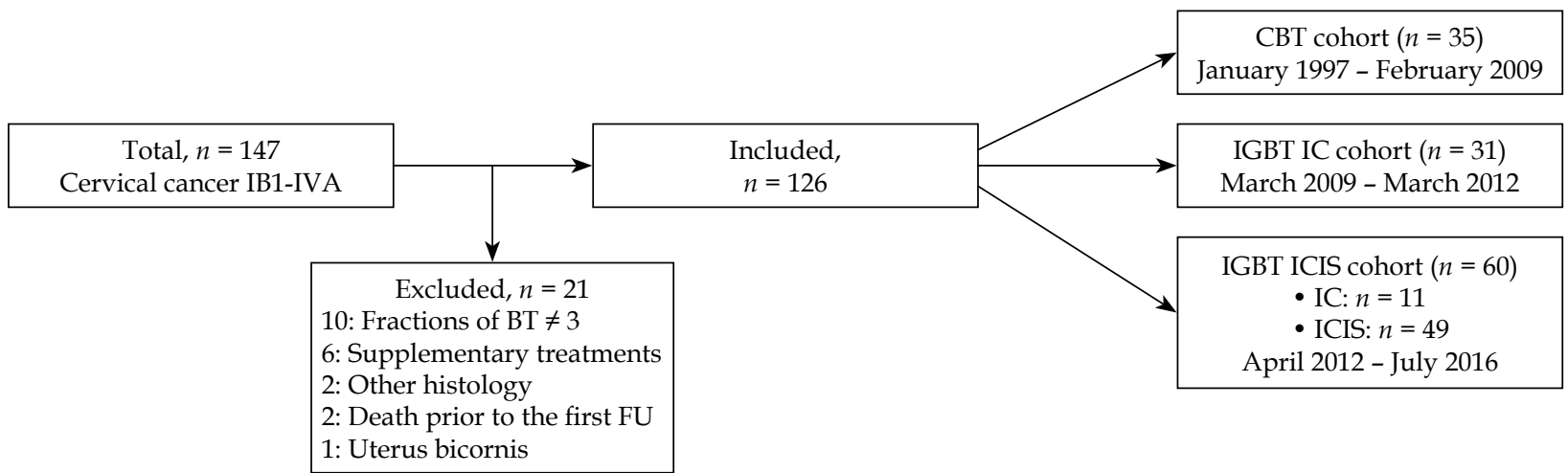

CBT - conventional brachytherapy, IGBT IC - image-guided brachytherapy intracavitary approach, IGBT ICIS - image-guided brachytherapy intracavitary approach (intentionally) with the use of interstitial needles

Fig. 1. Study design 
Table 1. Patient and tumor characteristics

\begin{tabular}{|c|c|c|c|c|}
\hline & CBT & IGBT IC & IGBT ICIS & $p$ value \\
\hline \multirow[t]{2}{*}{ Median age, year (range) } & $68(28-91)$ & $50(28-77)$ & $57(37-83)$ & 0.02 \\
\hline & $N(\%)$ & $N(\%)$ & $N(\%)$ & \\
\hline Groups & & & & 0.19 \\
\hline FIGO I & $12(34)$ & $5(16)$ & $13(22)$ & \\
\hline FIGO II & $16(46)$ & $19(61)$ & $26(43)$ & \\
\hline FIGO III + IV & $7(20)$ & $7(23)$ & $21(35)$ & \\
\hline Histology & & & & 0.60 \\
\hline Squamous cell carcinoma & $31(89)$ & $25(81)$ & $53(88)$ & \\
\hline Adenocarcinoma & $4(11)$ & $6(19)$ & $6(10)$ & \\
\hline Not specified & $0(0)$ & $0(0)$ & $1(2)$ & \\
\hline Nodal involvement & & & & 0.17 \\
\hline Yes & $10(29)$ & $16(52)$ & $27(45)$ & \\
\hline EBRT & & & & $<0.001$ \\
\hline 3D CRT (45 Gy) & $35(100)$ & $19(61)$ & $0(0)$ & \\
\hline IMRT (50 Gy) & $0(0)$ & $12(39)$ & $60(100)$ & \\
\hline Concurrent therapy & & & & $<0.001$ \\
\hline Chemotherapy & $20(57)$ & $28(90)$ & $57(95)$ & \\
\hline Hyperthermia & $1(3)$ & $1(3)$ & $1(2)$ & \\
\hline None & $14(40)$ & $2(7)$ & $2(3)$ & \\
\hline
\end{tabular}

CBT - conventional brachytherapy, IGBT IC - image-guided brachytherapy intracavitary approach, IGBT ICIS - image-guided brachytherapy intracavitary approach (intentionally) with the use of interstitial needles, 3D CRT - three-dimensional conformal radiation therapy, IMRT-intensity-modulated radiation therapy

A

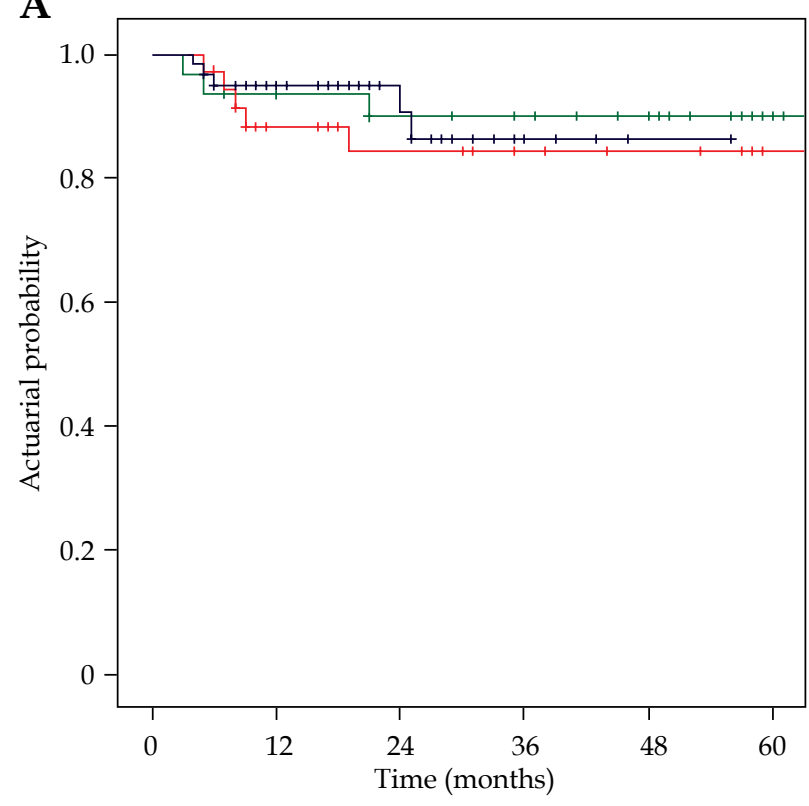

$\begin{array}{rcccc} & \text { Events } & N & 1 y & 3 y \\ \text { CBT } & 5 & 35 & 88 \% & 84 \% \\ \text { IGBT IC } & 3 & 31 & 94 \% & 90 \% \\ \text { IGBT ICIS } & 5 & 60 & 95 \% & 86 \% \\ p \text {-value } & 0.75 & & & \end{array}$

B

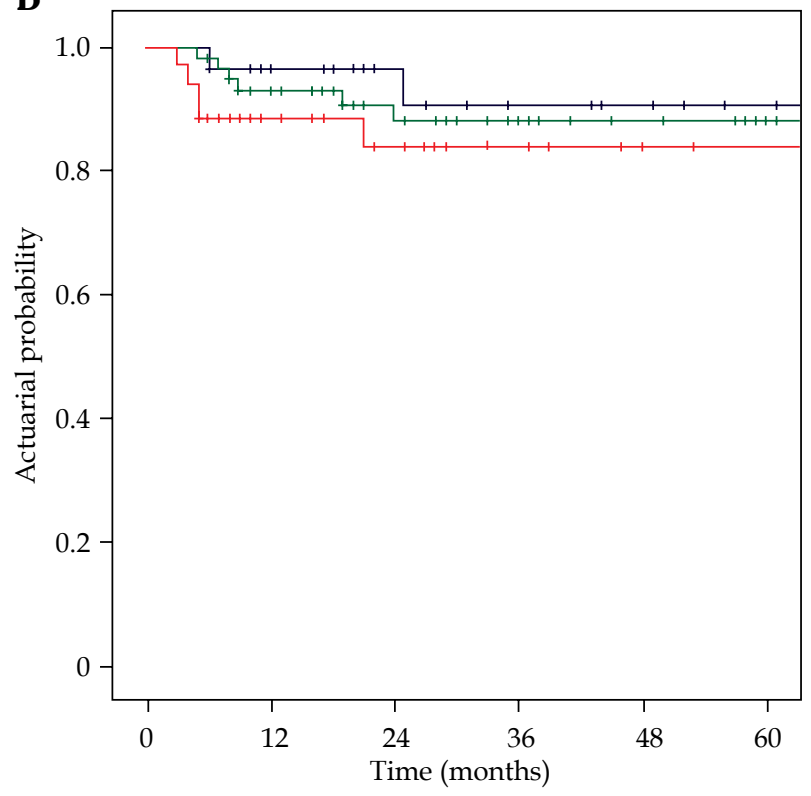

$\begin{array}{rcccc} & \text { Events } & N & 1 y & 3 y \\ \text { Group I } & 2 & 30 & 97 \% & 91 \% \\ \text { Group II } & 6 & 61 & 93 \% & 88 \% \\ \text { Group III } & 5 & 35 & 87 \% & 84 \% \\ p \text {-value } & 0.52 & & & \end{array}$

Fig. 2. A) Actuarial Kaplan-Meier estimates for one- and three-year local control (LC) per treatment cohort. CBT - conventional brachytherapy, IGBT IC - image-guided brachytherapy intracavitary approach, IGBT ICIS - image-guided brachytherapy intracavitary approach (intentionally) with the use of interstitial needles. B) Actuarial Kaplan-Meier estimates for one- and three-year local control (LC) per FIGO group 
A

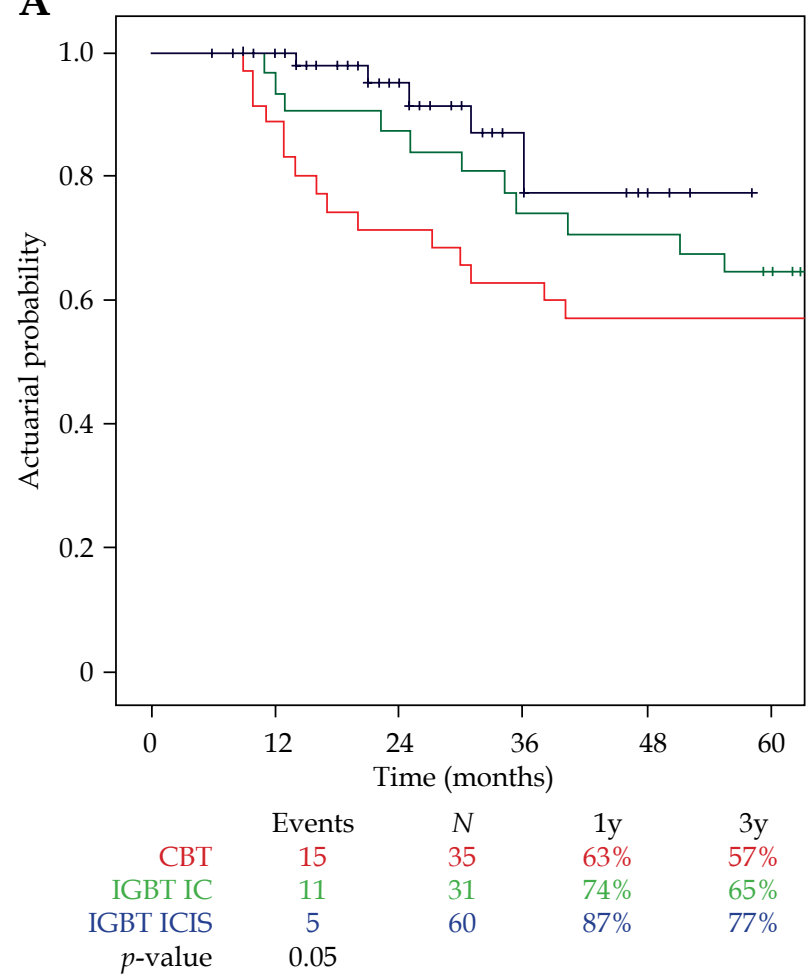

B

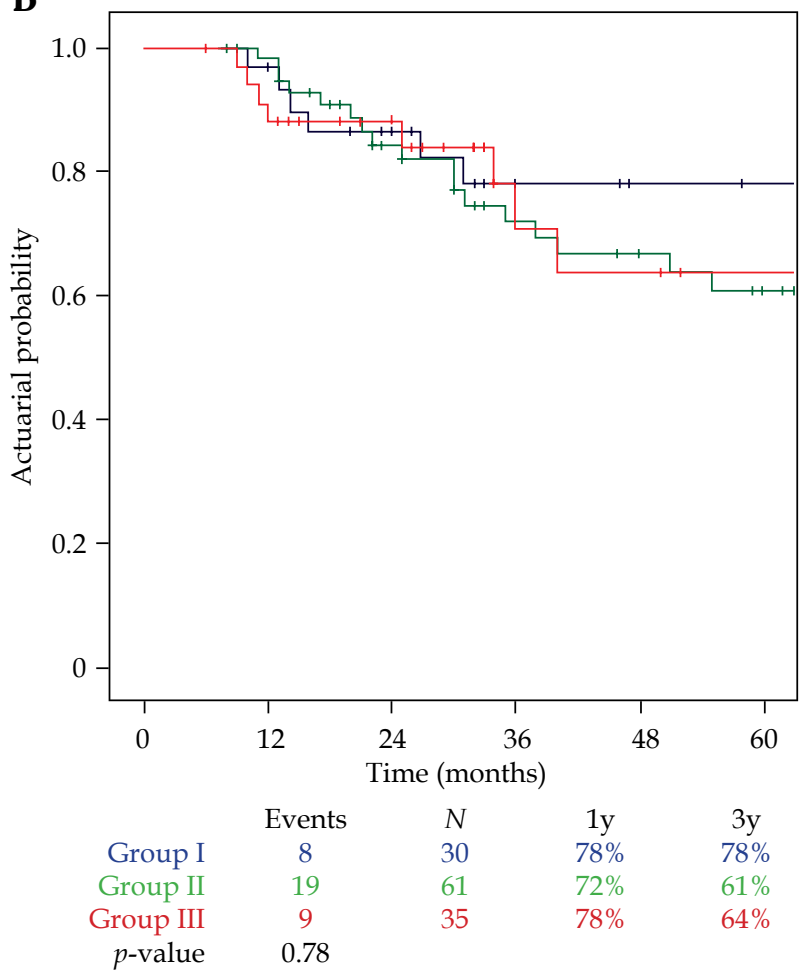

Fig. 3. A) Actuarial Kaplan-Meier estimates for three- and five-year overall survival (OS) per treatment cohort. CBT - conventional brachytherapy, IGBT IC - image-guided brachytherapy intracavitary approach, IGBT ICIS - image-guided brachytherapy intracavitary approach (intentionally) with the use of interstitial needles. B) Actuarial Kaplan-Meier estimates for three- and five-year overall survival (OS) per FIGO group

resulting in a three- and five-year overall survival of $75 \%$ and $66 \%$, and a three- and five-year cancer-specific survival of $82 \%$ and $77 \%$, respectively. A trend for a difference in overall survival was observed between the three treatment cohorts $(p=0.05$, Figure 3A). Overall survival was comparable in the FIGO groups $(p=0.78$, Figure $3 \mathrm{~B})$.

\section{Brachytherapy characteristics}

The potential benefit of interstitial needles was further analyzed in 27 patients, who had been treated without needles in the first fraction and with needles in the second fraction of BT. No difference in HR-CTV volume was observed between the first and second fraction $(p=0.78$, Table 2). A significant increase between fraction 1 and 2 was observed in the mean HR-CTV $D_{90}\left(E_{2}\right)$, which rose from 9.9 to $11.3 \mathrm{~Gy}(p<0.01)$, and in IR-CTV $\mathrm{D}_{90}\left(\mathrm{EQD}_{2}\right)$, which increased from 4.8 to $5.5 \mathrm{~Gy}(p<0.001)$. Also, a statistically significant difference was found in $\mathrm{D}_{2 \mathrm{cc}}$ bladder $\left(\mathrm{EQD}_{2}\right)$ dose, showing an average reduction from 14.7 to $10.9 \mathrm{~Gy}(p<0.01$, Table 2$)$.

\section{Late toxicity}

Grade 3 and 4 adverse events were observed in 17 of the 126 included patients (Table 3). One patient of the CBT cohort and one patient in the IGBT cohort had a rectovaginal fistula, which was treated with hyperbaric oxygen therapy (HOT). HOT was indicated in a patient of the IGBT cohort with chronic rectal and vaginal bleed-
Table 2. Impact of the use of interstitial needles on clinical tumor volume (CTV) dose and on dose to organs at risk (OAR) in 27 patients treated without needles in the first fraction and with needles in the second fraction of brachytherapy

\begin{tabular}{lccc} 
& $\begin{array}{c}\text { Dose fraction } \\
\text { 1 w/o needles }\end{array}$ & $\begin{array}{c}\text { Dose fraction } \\
\text { 2 w needles }\end{array}$ & $p$ value \\
\hline HR-CTV volume $\mathrm{cm}^{3}$ & $31.0 \pm 14.7$ & $31.6 \pm 14.5$ & 0.71 \\
\hline HR-CTV $D_{90}$ & $9.9 \pm 2.7$ & $11.3 \pm 1.8$ & 0.008 \\
\hline IR-CTV $D_{90}$ & $4.8 \pm 1.0$ & $5.5 \pm 0.9$ & 0.001 \\
\hline Bladder $D_{2 c c}$ & $14.7 \pm 5.9$ & $10.9 \pm 3.4$ & 0.002 \\
\hline Rectum $D_{2 c c}$ & $5.9 \pm 3.2$ & $5.4 \pm 2.6$ & 0.53
\end{tabular}

Mean $\pm S D$ for $H R-C T V D_{90}, I R-C T V D_{90}, D_{2 c c}$ bladder and $D_{2 c c}$ rectum. All doses are calculated in equi-effective dose (EQD2 10 for tumor and $E Q D 2_{3}$ for $O A R$ )

ing. Two patients had chronic urinary incontinence, and nerve stimulation was administered in one patient treated in the IGBT cohort. Overall, fewer adverse events were observed in the IGBT groups than in the CBT group (12\% vs. $17 \%)$, but the difference was not statistically significant $(p=0.31)$.

\section{Discussion}

The aim of this study was to compare the impact of 2D BT (CBT) and of 3D BT with and without interstitial 
Table 3. Adverse events as a result of brachytherapy in different cohort groups, conventional brachytherapy (CBT) vs. image-guided brachytherapy (IGBT)

\begin{tabular}{lccc} 
Adverse events & Total & CBT & IGBT IC + ICIS \\
\cline { 2 - 4 } & $n(\%)$ & $n(\%)$ & $n(\%)$ \\
\hline Number & 126 & 35 & 91 \\
\hline Grade 3/4 late adverse events crude rate & $17(13)$ & $6(17)$ & 11 (12) \\
\hline Rectovaginal fistula & 2 & 1 & 0 \\
\hline Vesicovaginal fistula & 1 & 1 & 1 \\
\hline Chronic urine incontinence & 2 & 1 & 1 \\
\hline Chronic rectal and vaginal bleeding & 1 & 0 & 0 \\
\hline Chronic diarrhea & 1 & 1 & 3 \\
\hline Radiation enteritis & 4 & 1 & 3 \\
\hline Radiation proctitis & 4 & 0 & 2
\end{tabular}

IGBT IC - image-guided brachytherapy intracavitary approach, IGBT ICIS - image-guided brachytherapy intracavitary approach (intentionally) with the use of interstitial needles

needles (IGBT ICIS and IGBT IC) on local control, overall survival, and toxicity in patients with cervical cancer after treatment with primary radiation or chemoradiation. Furthermore, the effect of dose sculpting with interstitial needles was evaluated. We found a trend toward an improved local tumor control, increased overall survival, and a decrease in toxicity with the introduction of MRI-guided BT in cervical cancer treatment. The additional use of interstitial needles allowed higher conformity in dose distributions.

\section{Local control}

The overall local control at three years was $88 \%$, which is comparable with the local control in the literature (Table 4). For example, the local control was 91\% in the RetroEMBRACE, a large multicenter study in patients with cervical cancer treated with chemoradiation followed by IGBT [6]. The current single-center study showed an improved local control in IGBT cohorts compared to the CBT cohort, but the difference was not statistically significant $(p=0.75)$ (Figure 2A). A possible explanation for this is the relatively unbalanced distribution of FIGO stages in the treatment cohorts (Table 1). There were more patients with low FIGO stages in the CBT cohort than in the IGBT cohorts. This could be related to the underuse of PET-CT and MRI in the CBT cohort. Lower FIGO stage is associated with better local control and overall survival [6,19]. This may in part explain the small difference in outcome between the treatment groups. We hypothesize that advancements in the BT techniques have resulted in referral and treatment of more extensive tumors, and higher FIGO stages with a worse prognosis. Local control rates in the FIGO groups also showed a trend for a better local control in lower FIGO stages (Figure 2B), as was reported in other studies $[6,9,10,11]$.

\section{Overall survival}

The three- and five-year overall survival was $75 \%$ and $66 \%$, respectively. This data is consistent with three-year overall survival rates in the literature, which range from $68 \%$ to $94 \%$, and with reported five-year overall survival rates, which are around $65 \%$ (Table 4 ). The treatment groups differed in overall survival rate, which was lower in the CBT cohort than in the IGBT cohorts. It is possible that the lower overall survival rate in the CBT cohort was due to higher median age at diagnosis and lower use of chemotherapy in this cohort. With regard to FIGO stage, we observed a negative association between overall survival and higher FIGO stage, which is consistent with findings of previous studies $[6,9,10,11]$.

\section{Brachytherapy characteristics}

In this study, the use of IS allowed a better tumor coverage while better sparing organs at risk. This is shown by the increase of the HR-CTV $\mathrm{D}_{90}$ and the IR-CTV $\mathrm{D}_{90}$, and by the decrease of $\mathrm{D}_{2 \mathrm{cc}}$ bladder (Table 2), as was also shown in other studies $[10,11,15,16,29,30,31]$. We hypothesize that these findings may result in a reduction of local recurrence, a better overall survival and, in particular, less toxicity. In this study, we could not confirm these implications, which was probably due to the relatively short follow-up of IGBT ICIS and low number of cases and events.

\section{Late toxicity}

We found a crude overall grade three or four toxicity rate of $13 \%$, toxicity being 17 to $12 \%$ lower in the IGBT cohorts than in the CBT cohort. This finding is in line with outcomes of the STIC trial, which reported a toxicity decrease of $50 \%$ since the introduction of IGBT [7]. The reason of this findings may be a better dose conformity, resulting in a decrease of dose to OAR. Further de- 


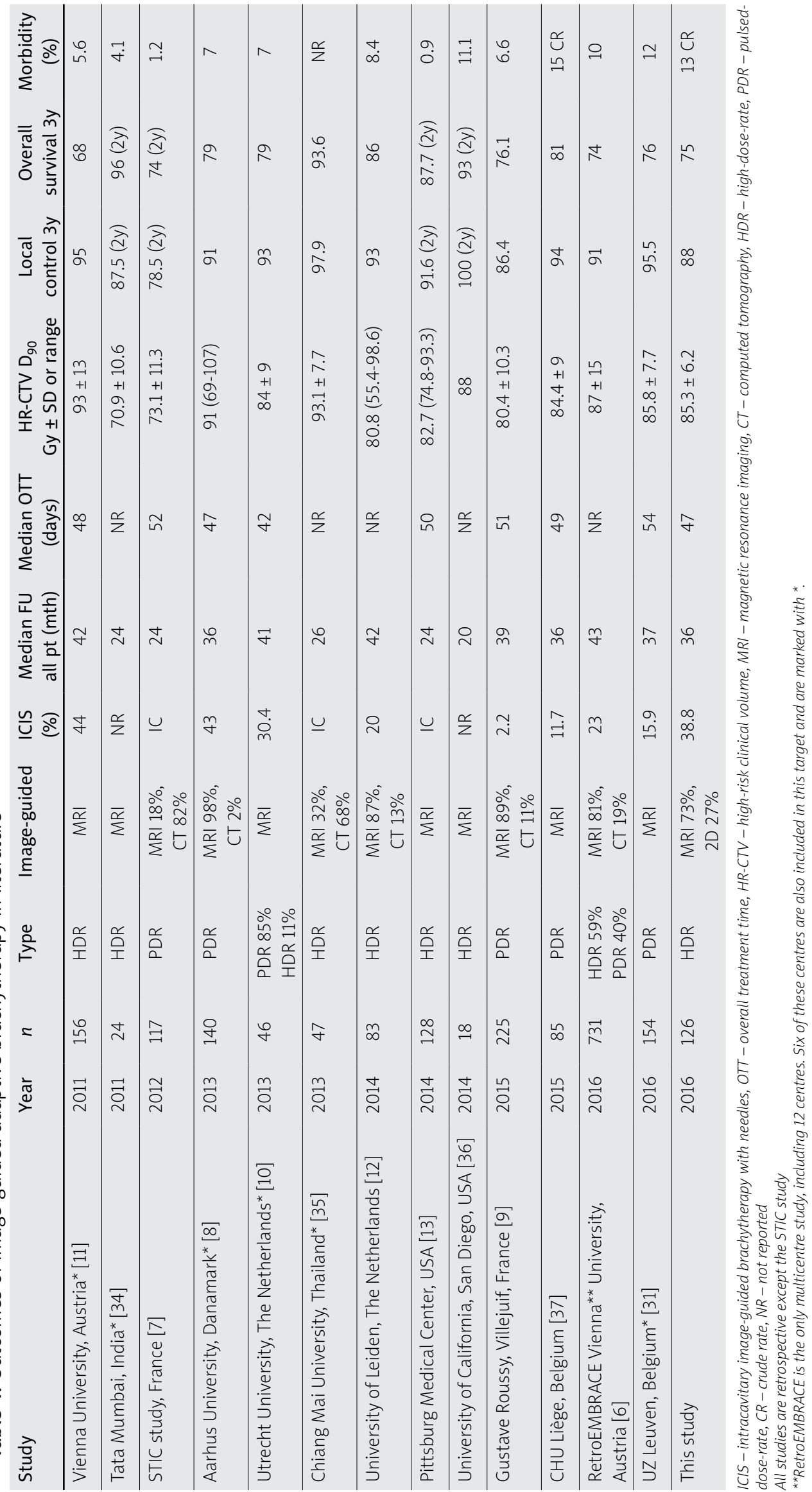


crease of the dose to OAR could be obtained by varying the bladder volume as reported by Siavashpour [32].

However, our results should be interpreted with caution because of the retrospective grading of toxicity and the shorter follow-up in the 3D IGBT cohorts, compared to the 2D CBT cohort. The limitations of our study mainly arise from the fact that our treatment cohorts differed with respect to the variables that influence local control and overall survival. Firstly, larger tumors (i.e., more advanced FIGO stages) were treated in the IGBT cohorts rather than in the CBT cohort, although good results were seen in all treatment cohorts. Secondly, the study had a low power, partly because of the low event rate in this limited number of patients, which precluded statistical correction for unbalanced variables. Finally, the follow-up in the IGBT ICIS cohort was short (20 months; range, 5 to 56 ), which potentially resulted in underrating toxicity. A larger number of patients and longer follow-up is needed to investigate whether the use of MRI-guided BT with interstitial needles will further allow dose escalation in large-volume tumors, and possible reduction in small-volume tumors, which may potentially result in additional toxicity decrease. This is the subject of the ongoing multicenter EMBRACE II study [33].

\section{Conclusions}

In this study, BT following radiation or chemoradiation for cervical cancer resulted in a three-year local control of $88 \%$ and an overall five-year survival of $75 \%$. BT techniques evolving from 2D (CBT) to 3D (MRI-guided IGBT) showed a trend towards an improved local tumor control, increased overall survival, and decreased toxicity. The introduction of interstitial needles led to increased tumor dose and lower dose to OAR. Although it could not be demonstrated that use of interstitial needles led to better local control, there was a trend towards reduced toxicity [34].

\section{Acknowledgements}

K.A.J. De Winter and F.L.A. Koppe are acknowledged for providing follow-up data of patients treated with EBRT at the Institute Verbeeten and referred for the brachytherapy boost to the study institute.

\section{Disclosure}

Authors report no conflict of interest.

\section{References}

1. Han K, Milosevic M, Fyles A et al. Trends in the utilization of brachytherapy in cervical cancer in the United States. Int J Radiat Oncol Biol Phys 2013; 87: 111-119.

2. Narayan K, Barkati $M$, van Dyk $S$ et al. Image-guided brachytherapy for cervix cancer: from Manchester to Melbourne. Expert Rev Anticancer Ther 2010; 10: 41-46.

3. Harkenrider MM, Alite F, Silva SR et al. Image-Based Brachytherapy for the Treatment of Cervical Cancer. Int J Radiat Oncol Biol Phys 2015; 92: 921-934.

4. Lindegaard JC, Tanderup K, Nielsen SK et al. MRI-guided 3D optimization significantly improves DVH parameters of pulsed-dose-rate brachytherapy in locally advanced cervical cancer. Int J Radiat Oncol Biol Phys 2008; 71: 756-764.

5. Tanderup K, Georg D, Pötter R et al. Adaptive management of cervical cancer radiotherapy. Semin Radiat Oncol 2010; 20: 121-129.

6. Sturdza A, Pötter R, Fokdal LU et al. Image guided brachytherapy in locally advanced cervical cancer: Improved pelvic control and survival in RetroEMBRACE, a multicenter cohort study. Radiother Oncol 2016; 120: 428-433.

7. Charra-Brunaud C, Harter V, Delannes M et al. Impact of 3D image-based PDR brachytherapy on outcome of patients treated for cervix carcinoma in France: results of the French STIC prospective study. Radiother Oncol 2012; 103: 305-313.

8. Lindegaard JC, Fokdal LU, Nielsen SK et al. MRI-guided adaptive radiotherapy in locally advanced cervical cancer from a Nordic perspective. Acta Oncol 2013; 52: 1510-1519.

9. Castelnau-Marchand P, Chargari C, Maroun P et al. Clinical outcomes of definitive chemoradiation followed by intracavitary pulsed-dose rate image-guided adaptive brachytherapy in locally advanced cervical cancer. Gynecol Oncol 2015; 139: 288-294.

10. Nomden CN, de Leeuw AA, Roesink JM et al. Clinical outcome and dosimetric parameters of chemo-radiation including MRI guided adaptive brachytherapy with tandem-ovoid applicators for cervical cancer patients: a single institution experience. Radiother Oncol 2013; 107: 69-74.

11. Pötter R, Georg P, Dimopoulos JC et al. Clinical outcome of protocol based image (MRI) guided adaptive brachytherapy combined with 3D conformal radiotherapy with or without chemotherapy in patients with locally advanced cervical cancer. Radiother Oncol 2011; 100: 116-123.

12. Rijkmans EC, Nout RA, Rutten IH et al. Improved survival of patients with cervical cancer treated with image-guided brachytherapy compared with conventional brachytherapy. Gynecol Oncol 2014; 135: 231-238.

13. Gill BS, Kim H, Houser CJ et al. MRI-guided high-dose-rate intracavitary brachytherapy for treatment of cervical cancer: the University of Pittsburgh experience. Int J Radiat Oncol Biol Phys 2015; 91: 540-547.

14. Tanderup K, Nielsen SK, Nyvang GB et al. From point A to the sculpted pear: MR image guidance significantly improves tumour dose and sparing of organs at risk in brachytherapy of cervical cancer. Radiother Oncol 2010; 94: 173-180.

15. Jürgenliemk-Schulz IM, Tersteeg RJ, Roesink JM et al. MRIguided treatment-planning optimisation in intracavitary or combined intracavitary/interstitial PDR brachytherapy using tandem ovoid applicators in locally advanced cervical cancer. Radiother Oncol 2009; 93: 322-330.

16. Otter S, Franklin A, Ajaz M et al. Improving the efficiency of image guided brachytherapy in cervical cancer. J Contemp Brachytherapy 2016; 8: 557-565.

17. Wang $X$, Li J, Wang $P$ et al. Image guided radiation therapy boost in combination with high-dose-rate intracavitary brachytherapy for the treatment of cervical cancer. J Contemp Brachytherapy 2016; 8: 122-127.

18. Pecorelli S. Revised FIGO staging for carcinoma of the vulva, cervix, and endometrium. Int J Gynaecol Obstet 2009; 105: 103-104.

19. Dutch National Guideline Cervical Cancer. Version 3.0 [www.oncoline.nl]. 2012: 93.

20. Franckena M, Lutgens LC, Koper PC et al. Radiotherapy and hyperthermia for treatment of primary locally advanced cervix cancer: results in 378 patients. Int J Radiat Oncol Biol Phys 2009; 73: 242-250.

21. van der Zee J, Franckena M. Cervical cancer. Int J Hyperthermia 2012; 28: 475-457. 
22. Lutgens L, van der Zee J, Pijls-Johannesma M et al. Combined use of hyperthermia and radiation therapy for treating locally advanced cervix carcinoma. Cochrane Database Syst Rev 2010; 3: CD006377.

23. Rivard MJ, Butler WM, DeWerd LA et al. Supplement to the 2004 update of the AAPM Task Group No. 43 Report. Medical Physics 2007; 34 (6Part1): 2187-2205.

24. Haie-Meder C, Pötter R, van Limbergen E et al. Recommendations from Gynaecological (GYN) GEC-ESTRO Working Group (I): concepts and terms in 3D image based 3D treatment planning in cervix cancer brachytherapy with emphasis on MRI assessment of GTV and CTV. Radiother Oncol 2005; 74: 235-245.

25. Pötter R, Haie-Meder C, van Limbergen E et al. Recommendations from gynaecological (GYN) GEC ESTRO working group (II): concepts and terms in 3D image-based treatment planning in cervix cancer brachytherapy-3D dose volume parameters and aspects of 3D image-based anatomy, radiation physics, radiobiology. Radiother Oncol 2006; 78: 67-77.

26. Hellebust TP, Kirisits C, Berger D et al. Recommendations from Gynaecological (GYN) GEC-ESTRO Working Group: considerations and pitfalls in commissioning and applicator reconstruction in 3D image-based treatment planning of cervix cancer brachytherapy. Radiother Oncol 2010; 96: 153-160.

27. Dimopoulos JC, Petrow P, Tanderup K et al. Recommendations from Gynaecological GEC-ESTRO Working Group (IV): Basic principles and parameters for MR imaging within the frame of image based adaptive cervix cancer brachytherapy. Radiother Oncol 2012; 103: 113-122.

28. Common Terminology Criteria for Adverse Events (CTCAE). Version 4.0. Available from: https://evs.nci.nih.gov/ftp1/CTCAE/CTCAE_4.03_2010-06-14_QuickReference_8.5x11.pdf.

29. Nomden CN, de Leeuw AA, Moerland MA et al. Clinical use of the Utrecht applicator for combined intracavitary/interstitial brachytherapy treatment in locally advanced cervical cancer. Int J Radiat Oncol Biol Phys 2012; 82: 1424-1430.

30. Fokdal L, Sturdza A, Mazeron R et al. Image guided adaptive brachytherapy with combined intracavitary and interstitial technique improves the therapeutic ratio in locally advanced cervical cancer: Analysis from the retroEMBRACE study. Radiother Oncol 2016; 120: 434-440.

31. Ribeiro I, Janssen H, De Brabandere $\mathrm{M}$ et al. Long term experience with 3D image guided brachytherapy and clinical outcome in cervical cancer patients. Radiother Oncol 2016; 120: 447-454.

32. Siavashpou Z, Aghamiri M, Jaberi R et al. Optimum organ volume ranges for organs at risk dose in cervical cancer intracavitary brachytherapy. J Contemp Brachytherapy 2016; 8: 135-142.

33. Tanderup K, Pötter R, Lindegaard JC et al. EMBRACE-II. Image guided intenstity modulated External beam radiochemotherapy and MRI based adaptive BRAchytherapy in locally advanced CErvical cancer 2015. Study protocol. p. 133.

34. Mahantshetty U, Swamidas J, Khanna N et al. Reporting and validation of gynaecological Groupe Euopeen de Curietherapie European Society for Therapeutic Radiology and Oncology (ESTRO) brachytherapy recommendations for MR image-based dose volume parameters and clinical outcome with high dose-rate brachytherapy in cervical cancers: a single-institution initial experience. Int J Gynecol Cancer 2011; 21: 1110-1116.

35. Tharavichitkul E, Wanwilairat S, Chakrabandhu S et al. Imageguided brachytherapy (IGBT) combined with whole pelvic intensity-modulated radiotherapy (WP-IMRT) for locally advanced cervical cancer: a prospective study from Chiang Mai University Hospital, Thailand. J Contemp Brachytherapy 2013; 5: 10-16.
36. Simpson DR, Scanderbeg DJ, Carmona R et al. Clinical Outcomes of Computed Tomography-Based Volumetric Brachytherapy Planning for Cervical Cancer. Int J Radiat Oncol Biol Phys 2015; 93: 150-157.

37. Lakosi F, de Cuypere M, Viet Nguyen P et al. Clinical efficacy and toxicity of radio-chemotherapy and magnetic resonance imaging-guided brachytherapy for locally advanced cervical cancer patients: A mono-institutional experience. Acta Oncol 2015; 54: 1558-1566. 